\title{
Manipulating hydrogen atoms using permanent magnets: Characterisation of a velocity-filtering guide
}

Cite as: Rev. Sci. Instrum. 90, 033201 (2019); https://doi.org/10.1063/1.5078573

Submitted: 26 October 2018 . Accepted: 13 February 2019 . Published Online: 07 March 2019

Jutta Toscano (D), Michal Hejduk (D), Henry C. McChee, and Brianna R. Heazlewood (D)

\section{ARTICLES YOU MAY BE INTERESTED IN}

\section{A magnetic guide to purify radical beams}

The Journal of Chemical Physics 149, 174201 (2018); https://doi.org/10.1063/1.5053656

Super-resolution imaging and field of view extension using a single camera with Risley prisms

Review of Scientific Instruments 90, 033701 (2019); https://doi.org/10.1063/1.5050833

A rate-doubled $10-\mathrm{GHz}$ fiducial comb generator for precision optical timing calibration Review of Scientific Instruments 90, 035103 (2019); https://doi.org/10.1063/1.5086159

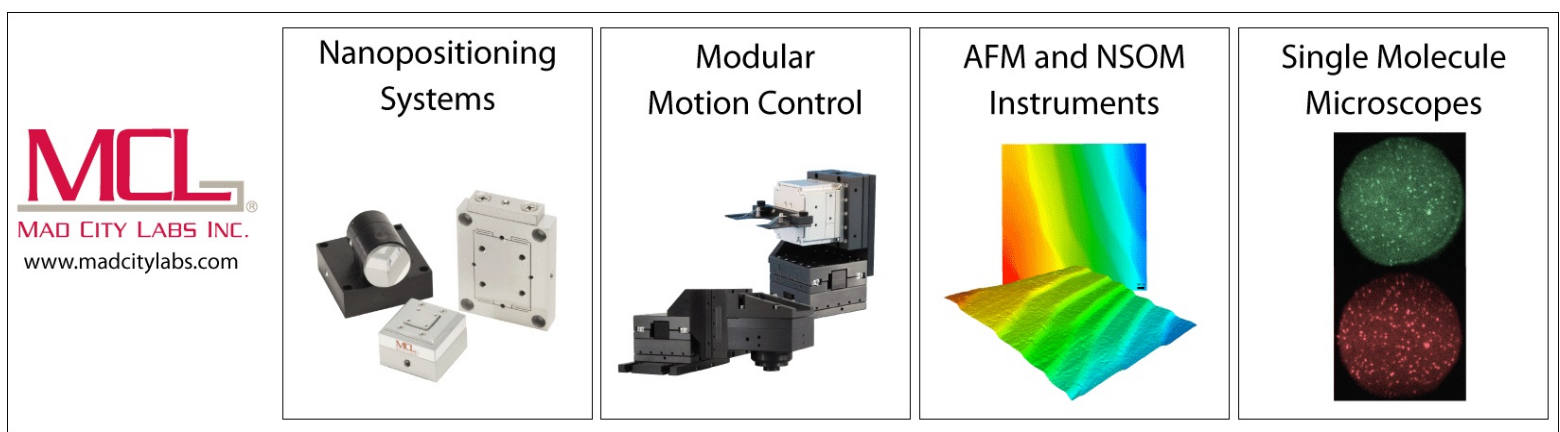




\title{
Manipulating hydrogen atoms using permanent magnets: Characterisation of a velocity-filtering guide
}

\author{
Cite as: Rev. Sci. Instrum. 90, 033201 (2019); doi: 10.1063/1.5078573 \\ Submitted: 26 October 2018 - Accepted: 13 February 2019 • \\ Published Online: 7 March 2019
}

Jutta Toscano, Michal Hejduk, (D) Henry G. McGhee, and Brianna R. Heazlewood (i)

\section{AFFILIATIONS}

Physical and Theoretical Chemistry Laboratory, Department of Chemistry, University of Oxford, South Parks Road, Oxford OX1 3QZ, United Kingdom

\begin{abstract}
A Halbach array composed of 12 permanent magnets in a hexapole configuration is employed to deflect hydrogen atoms as they exit a Zeeman decelerator. The ability to preferentially manipulate $\mathrm{H}$ atoms is very useful, as there are currently very few techniques that are appropriate for purifying a beam of $\mathrm{H}$ atoms from precursor molecules (such as molecular hydrogen or ammonia), seed gases, and other contaminant species. The extent to which hydrogen atoms are deflected by a single Halbach array when it is tilted or shifted off the main beam axis is characterised experimentally and interpreted with the aid of a simple mathematical model. A radical beam filter is subsequently introduced, where four Halbach arrays arranged in series serve to deflect $\mathrm{H}$ atoms away from the main beam axis and around skimming blades; all other components of the incoming beam are blocked by the blades and are thus not transmitted through the magnetic guide. The properties of the guide, as established by experimental measurements and complemented by detailed simulations, confirm that it is a highly effective beam filter-successfully generating a pure and velocity-selected beam of $\mathrm{H}$ atoms.
\end{abstract}

Published under license by AIP Publishing. https://doi.org/1 0.1 063/1.5078573

\section{INTRODUCTION}

The precise investigation of the dynamics of reactions involving unstable species such as radicals is a relatively unexplored field, owing to the challenges associated with studying these processes in the laboratory. It is notoriously difficult to prepare a pure, stateselected ensemble of radical reactants. Typical methods of forming radicals, such as through photodissociation, ${ }^{1}$ electron beam irradiation, ${ }^{2}$ or discharge methods, ${ }^{3,4}$ can also produce unwanted fragments and thus yield mixtures of precursor molecules, the radicals of interest, other dissociation products, and seed gases. Mass and velocity selection of negative ions followed by electron photodetachment is a viable method for generating a pure beam of radicals. 5,6 However, the radicals generated are generally fast-moving, and only species that readily accept a negative charge can be prepared in this way. As such, a generally applicable method that allows for the purification of a beam containing radicals (alongside other species) is needed.

Radicals can be separated from other components of a beam by exploiting their interaction with a magnetic field. The presence of an unpaired electron means that radicals possess an effective magnetic moment $\boldsymbol{\mu}$, which causes them to gain potential energy equal to $V_{B}=\boldsymbol{\mu} \cdot \mathbf{B}$ when they move into a magnetic field of strength B. Through the law of conservation of energy, this gain in potential energy is matched by an equivalent loss of kinetic energy. Zeeman decelerators, typically consisting of a series of solenoid electromagnet coils, make use of this behaviour; the solenoid-and thus the magnetic field-is switched off when the particles of interest reach a desired position within the coil. The force $F_{z}$ acting on a radical in a state with a positive magnetic moment along the field axis $\left(\mu_{z}>0\right.$, described as a low-field-seeker, LFS) and mass $m$, flying along the solenoid axis $z$, can be defined as

$$
F_{z}=m \ddot{z}=-\nabla_{z}\left[V_{B}(z, t)\right]=-\nabla_{z}\left[\mu_{z} B_{z}(z, t)\right] .
$$

While Zeeman deceleration is, in principle, applicable to all paramagnetic species, it is challenging to implement for species with low $\mu_{z} / m$, owing to the need for high field gradients and thus for high currents to be applied to the decelerator coils for tens of $\mu \mathrm{s}$, which can cause overheating of the device. Significant advances have been made toward improving the cooling efficiency of Zeeman decelerator solenoid coils, most recently by Cremers et al. ${ }^{7}$ who 


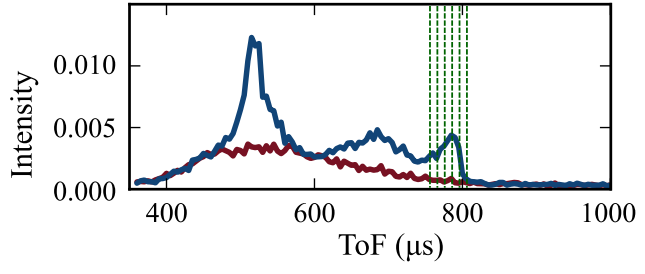

FIG. 1. Experimental Time-of-Flight (ToF) traces for a beam of hydrogen atoms detected after passing through the 12-stage Zeeman decelerator when no current is applied to the coils (the red line) and when magnetic fields are applied to decelerate a subset of the beam to a target final velocity of $205 \mathrm{~ms}^{-1}$ (the blue line). The green vertical dashed lines represent the scanning of the fully decelerated peak in order to record the intensity of the signal as a function of $y$ detection position (see text).

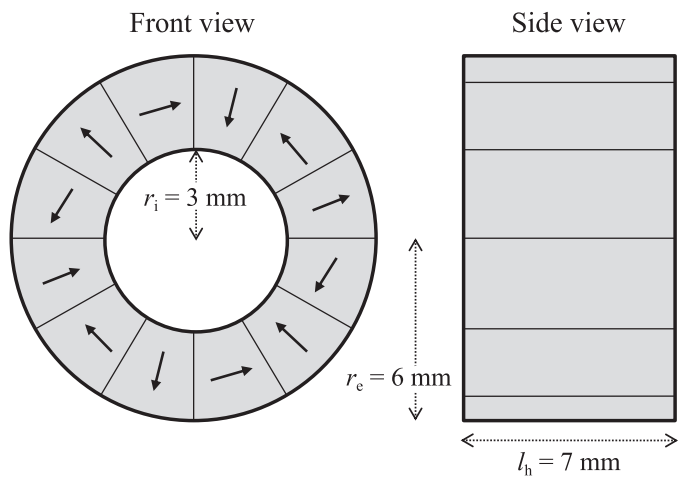

FIG. 2. Illustration of the dimensions of a Halbach array of permanent magnets in hexapolar configuration, as employed in this work. The Halbach array has remanence $B_{0}=1.3 \mathrm{~T}$, with arrows indicating the direction of the magnetic field (pointing from $\mathrm{S}$ to $\mathrm{N}$ ).

introduced a new coil design featuring direct contact between the solenoid and the cooling liquid. Others, such as Arimoto et al. ${ }^{8}$ and Truppe et al., ${ }^{9}$ chose to circumvent the switching problem by manipulating the magnetic moment $\mu_{z}(z=0)$ of particles in the magnetic field.

The Zeeman decelerator employed in this work (described previously in the work of Dulitz et al. ${ }^{10}$ ) is designed to decelerate $1 \mathrm{~s}{ }^{2} S_{1 / 2}$ hydrogen atoms in LFS quantum states. Hydrogen radicals are formed by photolysis (at $193 \mathrm{~nm}$ ) of a supersonic beam of ammonia seeded in krypton carrier gas and are decelerated to final velocities as low as $v_{z}=100 \mathrm{~ms}^{-1}$ by 12 solenoid coils with adjustable switching times. As the undecelerated beam has a modal velocity of approximately $v_{z}=500 \mathrm{~ms}^{-1}$, but a broad velocity range spanning several hundred $\mathrm{ms}^{-1}$, a significant proportion of particles are not sufficiently close to the "synchronous" particle in the initial velocity or position to be addressed by standard solenoid switching sequences. While we have tackled this issue by optimising the solenoid switching sequence so that more of the undecelerated particles are addressed, ${ }^{11}$ the output of the decelerator is still a mixture of decelerated, partially decelerated, and undecelerated $\mathrm{H}$ atoms (as shown in Fig. 1) as well as precursor materials and seed gases. One way of purifying such a beam would be to implement a mechanical shutter to physically block particles moving outside the target velocity, as has been used previously in our laboratory with a Stark decelerator. This is not an ideal solution for two reasons. In the first instance, the Zeeman decelerator in our laboratory is fairly short in length $(13 \mathrm{~cm})$, meaning that full spatial separation between the decelerated and undecelerated packets is not achieved by the end of the decelerator; a shutter alone would not effectively separate the decelerated and undecelerated particles unless it were placed some considerable distance after the final coil. Secondly, a shutter would also transmit all species travelling at the same speed as the decelerated radicals of interest (such as slow-moving seed gas or precursor molecules). A filter such as a bent magnetic guide using hexapole magnets in a Halbach array (HA) configuration has been proposed, ${ }^{12}$ but in such an approach, the target velocity range is determined by the radius of the bend and thus not easily varied.

In this work, we report a detailed characterisation of our new approach for the generation of a pure source of radicals with tuneable velocity: the approach utilises a magnetic guide composed of four Halbach arrays and two skimming blades, where only those radicals falling within a selected narrow velocity range are transmitted. ${ }^{13}$ A Halbach array (HA) is an arrangement of permanent magnets in a hexapole configuration (see Fig. 2), which augments the magnetic field in one region while minimising the field in another ${ }^{14}$ - there is a very strong magnetic field inside the array with weak magnetic fields beyond it. Halbach arrays have been previously employed for guiding neutral beams ${ }^{15}$ and to improve the transverse confinement of particles in between Zeeman deceleration stages. ${ }^{7}$ The first part of this manuscript focuses on describing how a radical beam is deflected with a single HA. The detailed experimental verification of the performance of a single array is critical for the design of the four-array filter or indeed for any other deflecting device seeking to use such arrays on radical beams. Two modes of deflection, as illustrated in Fig. 3-shifting in a vertical direction (along the $y$ axis) or tilting (in the $y z$ plane)-are compared to derive an optimal arrangement for a series of HAs to serve as an efficient and effective
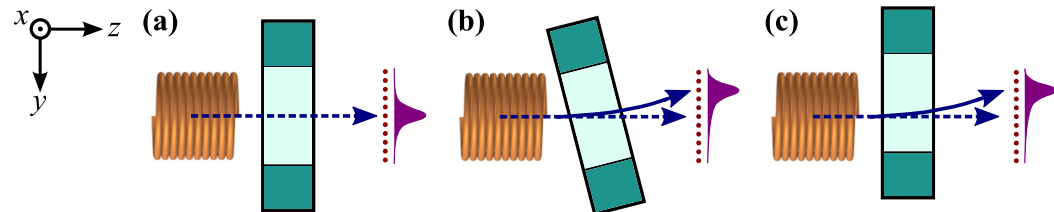

FIG. 3. Schematic representation of the relative position of the Halbach array (in green, side view) (a) when it is perfectly aligned, (b) tilted (in the yz plane), and (c) shifted (in the $y$ direction) with respect to the final decelerator coil (in orange). The blue arrows represent the trajectories of the deflected (solid lines) and undeflected (dashed lines) beams. The vertical red dots represent the detection positions probed in the characterization experiments for one Halbach array, and the schematic beam profiles (in purple) represent the (a) undeflected and (b and c) deflected intensity profiles obtained. 
beam filter. Measured beam deflections are compared with computer simulations and are discussed using a simple mathematical model. In the second part of the manuscript, the beam-filtering capabilities of the new optimal guide design are quantified by experiments and computer simulations.

\section{BEAM MANIPULATION BY A SINGLE HALBACH ARRAY}

In order to characterise the beam deflection achievable with one Halbach array, a 12-segment $\mathrm{NdFeB} \mathrm{HA}$ with remanence $B_{0}=1.3 \mathrm{~T}$, internal radius $r_{i}=3 \mathrm{~mm}$, external radius $r_{e}=6 \mathrm{~mm}$ and thickness $l_{h}$ $=7 \mathrm{~mm}$ is used. The array is mounted on a linear actuator coupled with a $z$ translation stage (to allow for linear movement parallel to the $y$ axis as well as rotation in the $y z$ plane) and positioned $6 \mathrm{~mm}$ beyond the last solenoid coil of the decelerator [Fig. 3(a)]. The Zeeman decelerator is operated using a deceleration sequence that slows down hydrogen atoms to a target final velocity of $205 \mathrm{~ms}^{-1}$, generating the Time-of-Flight (ToF) trace shown in Fig. 1. The transmitted beam is resonantly ionised via $(2+1)$ REMPI at $243 \mathrm{~nm}$; the detection laser beam propagates along the $x$ axis and is estimated from simulations to extend $0.8 \mathrm{~mm}$ in the $z$ dimension and $0.4 \mathrm{~mm}$ in the $y$ dimension, as reported previously. ${ }^{11}$ The resulting ions are accelerated by using a Wiley-MacLaren-type ToF mass spectrometer toward microchannel plates for detection.

The transmittance and deflection of particles as a function of the amount of tilt [Fig. 3(b)] or shift [Fig. 3(c)] of the Halbach array with respect to the last decelerator coil is investigated by detecting $\mathrm{H}$ atoms at a range of different positions along the $y$ axis. This enables one to build up a profile of the spatial distribution of $\mathrm{H}$ atoms after the Halbach array (purple profiles in Fig. 3). $\mathrm{H}$ atoms are detected by moving the ionisation laser vertically from $y=-5 \mathrm{~mm}$ to $y=+5 \mathrm{~mm}$ (with $y=0 \mathrm{~mm}$ corresponding to the decelerator axis) in steps of $1 \mathrm{~mm}$, as represented by the red dots in Fig. 3. At each detection position, the intensity of the signal from the decelerated peak is recorded at $10 \mu \mathrm{s}$ intervals over a range of $50 \mu \mathrm{s}$, as represented by

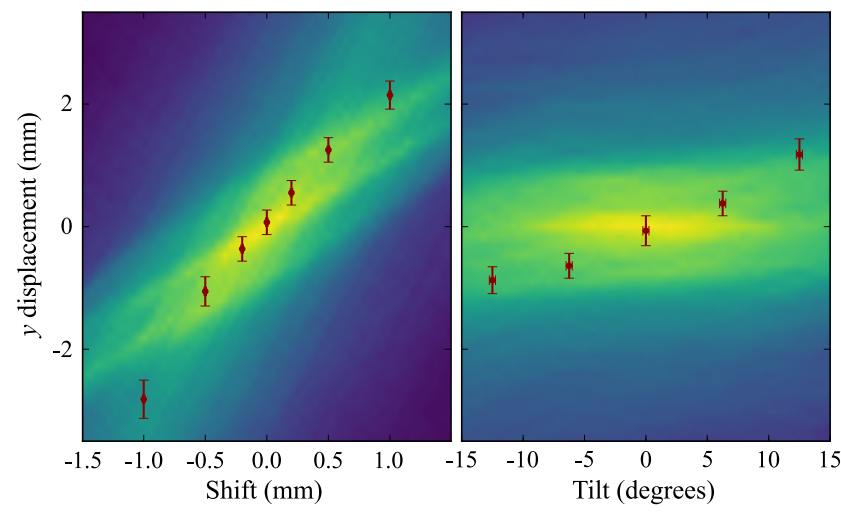

FIG. 4. Displacement of decelerated particles in the $y$ direction as a function of shift (left panel) and tilt (right panel) of one Halbach array. The simulated data are represented with intensity ranging from low (purple) to high (yellow). The experimental measurements are represented by red dots, with error bars indicating the respective uncertainties. the vertical dashed lines in Fig. 1. The integrated signal for each scan is subsequently plotted as a function of detection position along the $y$ axis, and a Gaussian profile is fit to the experimentally measured signal levels. This process is repeated for a range of tilting angles $\left(0.0^{\circ}, \pm 6.25^{\circ}, \pm 12.5^{\circ}\right)$ and shifting distances $(0.0 \mathrm{~mm}, \pm 0.2 \mathrm{~mm}$, $\pm 0.5 \mathrm{~mm}, \pm 1.0 \mathrm{~mm}$ ), where a tilt of $0.0^{\circ}$ and a shift of $0.0 \mathrm{~mm}$ correspond to the Halbach array being perfectly aligned with the last decelerator coil [Fig. 3(a)]. The maximum of each Gaussian and its uncertainty are shown as dots with respective error bars in Fig. 4, where displacement of the decelerated peak in the $y$ direction is plotted as a function of the amount of shift (left panel) and tilt (right panel). The intensity plots represent the predictions from threedimensional particle-trajectory simulations, in which four million particles emerging from the decelerator are flown through a tilted or shifted Halbach array ${ }^{1,13}$ (modelled using the Radia software package ${ }^{16-18}$ ). Tilting angles are simulated between $\pm 15^{\circ}$ in steps of $0.5^{\circ}$, whilst shifting distances are simulated between $\pm 1.5 \mathrm{~mm}$ in steps of $0.05 \mathrm{~mm}$.

\section{RESULTS AND DISCUSSION}

As can be seen from Fig. 4, increasing the absolute shifting distance of the Halbach array displaces the decelerated peak along $y$ more than increasing the absolute tilting angle of the array does, within the experimentally accessible tilting and shifting ranges. Additionally, the experiments indicate that tilting the HA disperses the decelerated beam more than shifting and therefore yields broader beam profiles. Indeed, the average full-width-at-halfmaximum measurements of the Gaussians fit to the experimental data are $7.5 \mathrm{~mm}$ for shifting and $9.4 \mathrm{~mm}$ for tilting. The simulated shifting behavior (Fig. 4, left panel) agrees well with the experimental measurements (red dots). However, the experimentally measured displacement induced by tilting is under-estimated by the simulation (right panel). This is attributed to the limitations of our model. For example, whenever a simulated particle encounters a surface (such as the HA), it is removed from the simulation. As the obstruction of the field of view of the particles is greater when the HA is tilted rather than shifted (see Fig. 5), more particles are likely to travel closer to the surfaces of the HA. Hence neglecting surface-particle interactions is expected to have a greater impact on the simulations of the tilted array. Nevertheless, in both the experimental measurements and the simulations, the maximum displacement of the beam achievable with a tilted HA is clearly lower than that achievable with a shifted HA, within the accessible tilting and shifting ranges.

The correlated distribution of the forward velocities, beam divergences, and radial positions of the particles in the beam $\left(\rho_{v_{z}}\right.$, $\rho_{\alpha}$, and $\rho_{r_{1}}$, respectively) is obtained from simulations of the particle kinetics following the Zeeman deceleration to a final target velocity of $205 \mathrm{~ms}^{-1}$ and is plotted in Fig. 6. As the Zeeman decelerator model reproduces the experimental ToF spectra very well, ${ }^{11}$ derived functions such as $\rho_{v_{z}}, \rho_{\alpha}$, and $\rho_{r_{1}}$ are used without further experimental verification. It can be seen from Fig. 6 that the beam entering the HA has a slightly wider beam radius than the HA inner radius $\left(r_{i}=3 \mathrm{~mm}\right)$ and a fairly uniform density within $r_{1} \in\left(0.0, r_{b}\right)$. The majority of particles travelling at the target velocity of $v_{z}=205 \mathrm{~ms}^{-1}$ follow trajectories that enable them to be transmitted through the HA; a small number of particles exhibit radii $r_{1}>r_{i}$ and are lost 
(a)

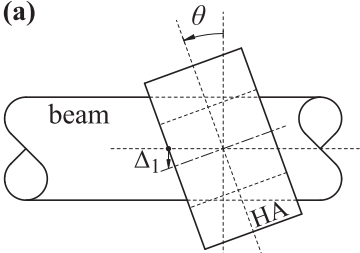

(b)

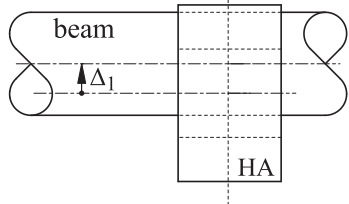

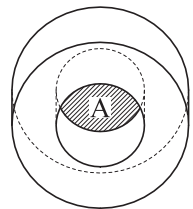

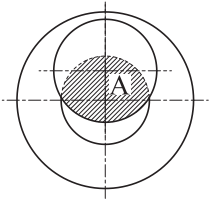

FIG. 5. Obstruction of the field of view $A$ by (a) tilting or (b) shifting the HA. Areas $A$ can be imagined as being a projection of a perfectly collimated particle beam on a screen behind the HA. Such a model is justifiable as the beam divergence $\alpha$ is low with respect to its physical dimensions.

from the beam as it passes through the HA. There is a strong correlation between the forward velocity $\left(v_{z}\right)$ and the divergence $(\alpha)$; particles with velocities around $300 \mathrm{~ms}^{-1}$ and $500 \mathrm{~ms}^{-1}$ exhibit the greatest divergence (not shown). This property is exploited in the design of a beam filter comprised of several HAs in series, as these faster-moving $\mathrm{H}$ atoms will be preferentially lost from the beam as it travels through the guide. The majority of the simulated $\mathrm{H}$ atoms follow trajectories that fall within the $r_{1} \in\left(0.0, r_{i}\right)$ acceptance limit of the HA, with high-radius/high-divergence trajectories representing only a small fraction of the ensemble.

For a qualitative understanding of the experimental data, it is beneficial to derive an analytical formula for the displacement of the beam by a single HA. Following the derivation given by Borodi ${ }^{19}$ and using a three-by-three matrix formalism for "misaligned" systems, ${ }^{20}$ it is possible to show that the hexapolar HA behaves like a thick lens with a ray transfer matrix,

$$
\boldsymbol{M}=\left(\begin{array}{ccc}
\cos (L \lambda) & \frac{\sin (L \lambda)}{\lambda} & \theta\left(L-\frac{\sin (L \lambda)}{\lambda}\right)+(1-\cos (L \lambda)) \Delta_{1} \\
-\lambda \sin (L \lambda) & \cos (L \lambda) & \theta(1-\cos (L \lambda))+\lambda \sin (L \lambda) \Delta_{1} \\
0 & 0 & 1
\end{array}\right),
$$

with parameters and variables defined in Table I and illustrated in Fig. 7. This formula is valid for small deflection angles and axial magnetic field gradients $\nabla_{z} \mathbf{B}$, which holds only in the vicinity of the lens axis.

Continuing with this analogy (whereby $\mathrm{H}$ atoms deflected by a $\mathrm{HA}$ are treated in the same manner as rays of light manipulated by a lens), upon exiting the $\mathrm{HA}$, a ray $\left(r_{1}, \alpha, 1\right)^{\mathrm{T}}$ is displaced to a position

$$
\begin{aligned}
r_{o}= & \Delta_{1}[\lambda l \sin (\lambda L)-\cos (\lambda L)+1] \\
& +\theta\left\{l[1-\cos (\lambda L)]-\frac{\sin (\lambda L)}{\lambda}+L\right\} \\
& +r_{1}[\cos (\lambda L)-\lambda l \sin (\lambda L)]+\frac{\alpha[\lambda l \cos (\lambda L)+\sin (\lambda L)]}{\lambda}
\end{aligned}
$$

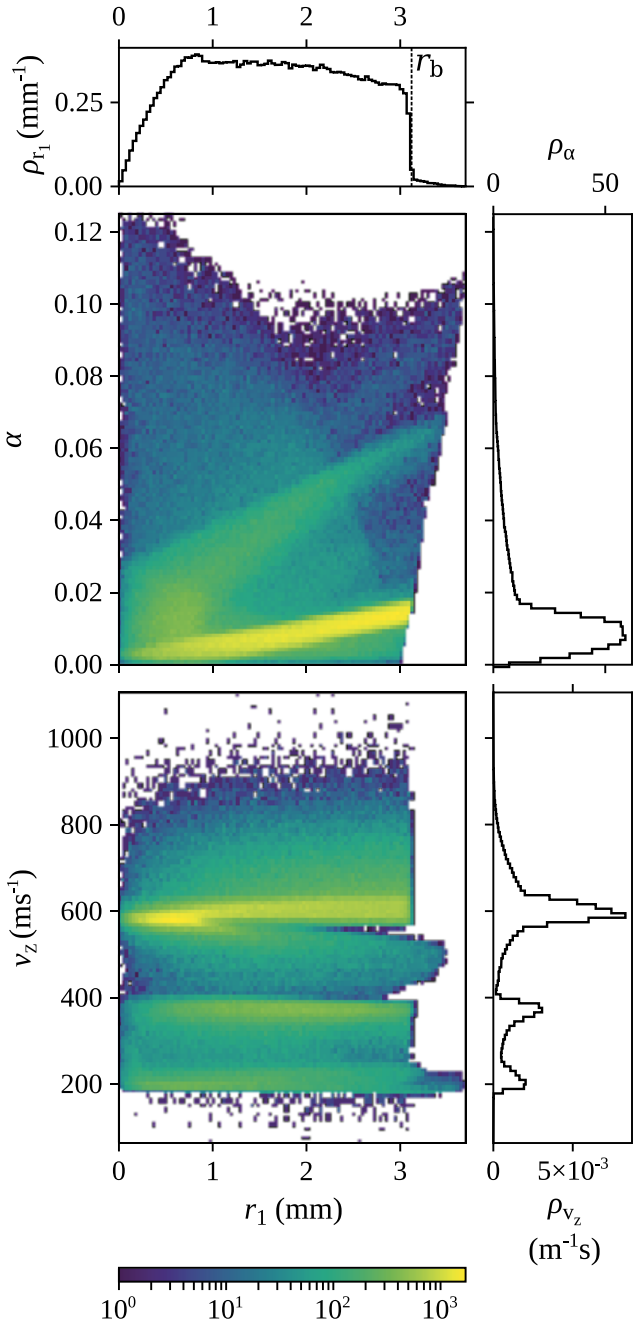

FIG. 6. Dependencies of the simulated forward velocity $v_{z}$ and beam divergence $\alpha$ distributions on the radial position $r_{1}$, for a beam decelerated to a target velocity of $205 \mathrm{~ms}^{-1}, 6 \mathrm{~mm}$ beyond the exit of the decelerator (corresponding to the $z$-axis position of the entrance to the $\mathrm{HA}$ ). Distributions integrated over the radial positions ( $\rho_{\alpha}$ for the divergence and $\rho_{v_{z}}$ for the forward velocity) and the $r_{1}$ distribution $\left(\rho_{r_{1}}\right)$ are plotted as projections along the respective axes. The simulations include $10^{6}$ particles, with relative intensity defined by the colour bar.

on a screen (i.e., to a position on the $x y$ plane) at distance $l$ from the HA. ${ }^{21}$ This is a linear function of $\Delta_{1}$, and, indeed, the measured dependence of the beam deflection as a function of the shift along the $y$ axis in Fig. 4 is linear. In the case of tilting with respect to the centre of the HA, $r_{o}$ becomes a combination of the $1 \times \theta$, $\sin (\theta)$, and $\tan (\theta)$ functions. As the incoming beam radius is greater than the inner radius of the HA, $r_{b}>r_{i}$, part of the incoming beam is physically blocked when the HA is shifted or tilted away from the central axis of the decelerator. In other words, the beam's field of view $A$ (as defined in Fig. 5) is reduced as the HA is shifted or tilted. 
TABLE I. Variables introduced in the analytical expression describing beam deflection by a shifted or tilted HA.

\begin{tabular}{|c|c|c|}
\hline Symbol & Definition & Description \\
\hline$L$ & Lens width & Effective length of the HA lens, $L \sim l_{h}$ \\
\hline$\lambda$ & Spatial frequency & $\lambda^{2}=2 \mu B_{r} /\left(m v_{z}^{2} r_{0}^{2}\right)$ \\
\hline$\Delta_{1}$ & Vertical displacement & Displacement of the centre of the HA entrance from $y=0$ \\
\hline$\theta$ & Tilting angle & Tilting angle of the HA measured from the $y$ axis \\
\hline$\mu$ & Magnetic moment & Effective magnetic moment of the radical \\
\hline$v_{z}$ & Forward velocity & Velocity component of the radical along the $z$ axis \\
\hline$B_{r}$ & Radial magnetic field & $B_{r}=B_{0}\left(r / r_{i}\right)^{2}$, assuming cylindrical coordinates and ignoring fringe effects at $r_{i}$ \\
\hline$B_{z}$ & Axial magnetic field & Magnetic field along the $z$ axis \\
\hline$B_{0}$ & Central magnetic field & Magnetic field at the centre of the HA \\
\hline$r_{i}$ & HA radius & Inner radius of the $\mathrm{HA}$ \\
\hline$\alpha$ & Divergence & Input beam angle measured from the optical axis anti-clockwise (see Fig. 7) \\
\hline$r_{1}$ & Input beam height & Vertical position of an $\mathrm{H}$ atom, with respect to the $z$ axis \\
\hline$l$ & Screen distance & Detection screen distance, measured from the rear face of the HA \\
\hline$m$ & Particle mass & \\
\hline
\end{tabular}

With the aid of this analytical expression-alongside detailed computer simulations-one can begin to interpret the experimentally measured deflection resulting from tilting or shifting a single HA. In designing a radical beam filter, it is necessary to optimise transmission of the desired species (in this case, $\mathrm{H}$ atoms travelling at a selected velocity) whilst hindering the passage of all other components of the beam, which can be achieved by using several arrays in series. As demonstrated above, placing a shifted HA in the beam deflects the decelerated peak more, as well as dispersing it less, than a tilted HA. Designing a beam filter with shifted (rather than tilted) HAs also has practical advantages as it is more straightforward to implement. For these reasons, shifted Halbach arrays are chosen as the optimal deflection method in the design of the magnetic guide.

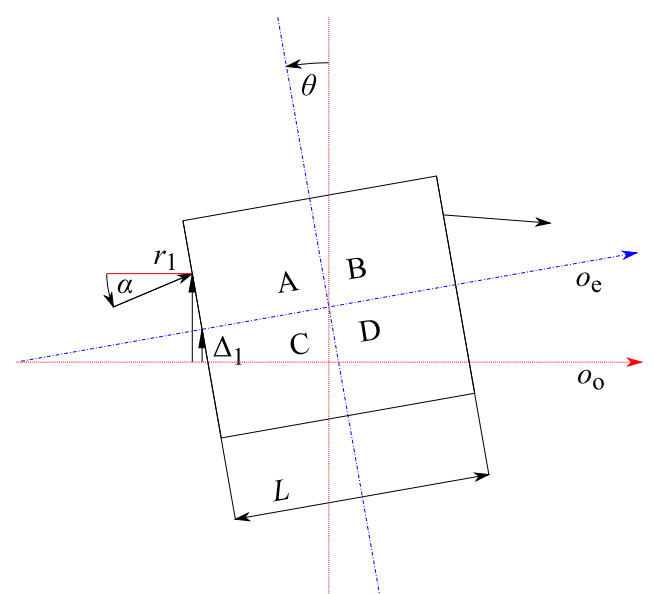

FIG. 7. Coordinate system for an optical element that displaces a beam, as described by an $A B C D$ ray transfer matrix, adapted from Siegman. ${ }^{20}$ The symbols $O_{e}$ and $o_{0}$ denote the element and optical axes, respectively.

\section{THE MAGNETIC GUIDE}

A magnetic guide with four HAs arranged in series is utilised to filter the hydrogen atoms travelling at the desired velocity from the rest of the beam. In this way, the beam is deflected away from the decelerator axis (by the first HA), collimated (by the second HA), deflected back onto the decelerator axis (by the third HA), and collimated a final time (by the fourth HA). Simulations evaluating the effect of remanence $B_{0}$, internal radius $r_{i}$, external radius $r_{e}$, and thickness $l_{h}$ of the arrays on focal length indicate that Halbach arrays with $B_{0}=1.3 \mathrm{~T}, r_{i}=4 \mathrm{~mm}, r_{e}=7 \mathrm{~mm}$, and $l_{h}=5 \mathrm{~mm}$ are suitable for manipulating $\mathrm{H}$ atoms with final velocities between 125 and $200 \mathrm{~ms}^{-1}$ - the target range for our Zeeman decelerator. As such, Halbach arrays with these properties are chosen for the guide. Skimming blades are used to physically block the undeflected components of the beam. A schematic representation of the magnetic guide is shown in Fig. 8, with the passage of $\mathrm{H}$ atoms travelling at

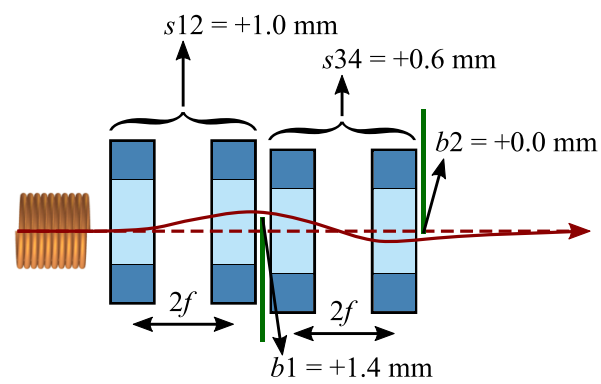

FIG. 8. Schematic depiction of the optimal magnetic guide design. The labels $s 12$ and $s 34$ indicate the vertical shift of the first two Halbach arrays and of the second two Halbach arrays, respectively; $b 1$ corresponds to the $y$ position of the top end of the first blade with $b 2$ the $y$ position of the bottom end of the second blade. $f$ is the focal length of the Halbach array. The passage of the transmitted particles is shown by the solid red line, with the dashed red line indicating the decelerator $(z)$ axis. 


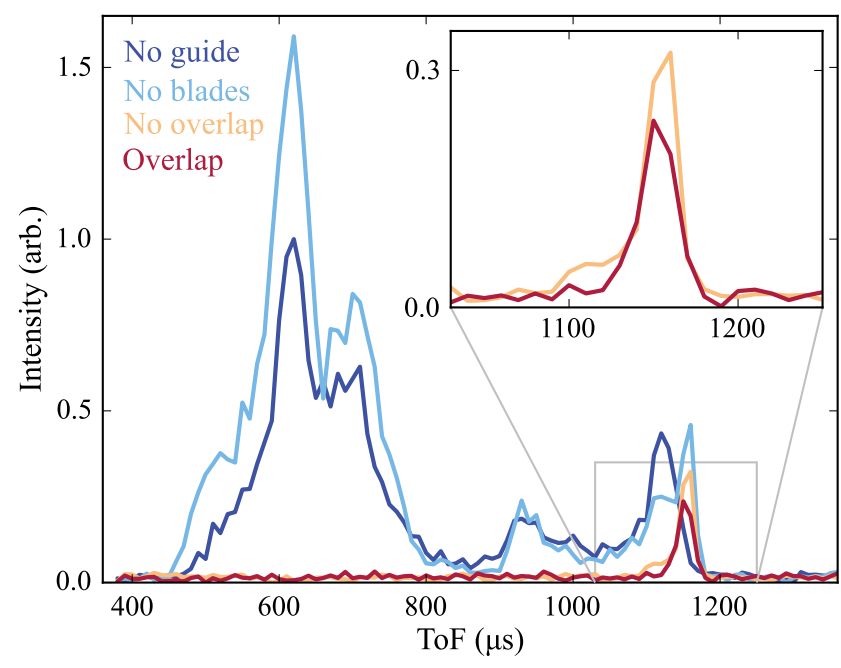

FIG. 9. Experimental ToF traces for a beam of hydrogen atoms with a target final velocity of $200 \mathrm{~ms}^{-1}$, detected after passing through the 12-stage Zeeman decelerator, with and without the magnetic guide present. ToF traces are recorded with no guide present (dark blue, labelled "No guide"), with the four Halbach arrays of the magnetic guide in place but with the skimming blades retracted such that there is an $8 \mathrm{~mm}$ gap between them (light blue, labelled "No blades," $b 1=-4.0 \mathrm{~mm}$ and $b 2=+4.0 \mathrm{~mm}$ ), with the blades positioned at the beam axis (orange trace, labelled "No overlap," $b 1=b 2=0.0 \mathrm{~mm}$ ), and with the blades at their optimised positions (red trace, labelled "Overlap," $b 1=+1.4 \mathrm{~mm}$ and $b 2=0.0 \mathrm{~mm}$ ). The inset shows only the "No overlap" and "Overlap" traces. the target velocity illustrated. An important feature of the blades is that they penetrate the beam axis, breaking the cylindrical symmetry such that there is no direct line-of-sight through the magnetic guide. This design feature ensures that only particles moving at the target velocity reach the detector. The skimming blades are optimised to a vertical shift of $y=b 1=+1.4 \mathrm{~mm}$ for the first blade and $y=b 2=0.0 \mathrm{~mm}$ for the second blade. This geometry balances the need for a narrow velocity distribution and maintaining high transmission through the guide. As the overlap of the blades is a tuneable feature of the design, one could amend their positions to achieve higher transmission or narrower velocity resolution if desired.

To quantify the effectiveness of the guide, four experimental ToF spectra are recorded: without the guide, with the guide in place but with the skimming blades retracted, with the guide in place and the skimming blades positioned at $b 1=b 2=y=0.0 \mathrm{~mm}$, and with all elements of the guide in their optimised positions. As Fig. 9 illustrates, once the blades are introduced, only the last peak in the ToF spectra is present-corresponding to the slowest-moving hydrogen atoms; undecelerated and partially decelerated species are blocked by the blades. This is explained pictorially in Fig. 10, where the simulated trajectories of hydrogen atoms travelling above the target velocity $\left(>210 \mathrm{~ms}^{-1}\right)$ are plotted. Overlapping the guide blades is essential for obtaining a narrow velocity distribution in the resulting beam. The intensity of the peak containing the guided decelerated particles in the ToF trace (at approximately $1160 \mu \mathrm{s}$ ) is reducedand the small higher-velocity shoulder (at $1110 \mu \mathrm{s}$ ) to this peak is absent-when the blades are overlapped (the inset of Fig. 9). This is
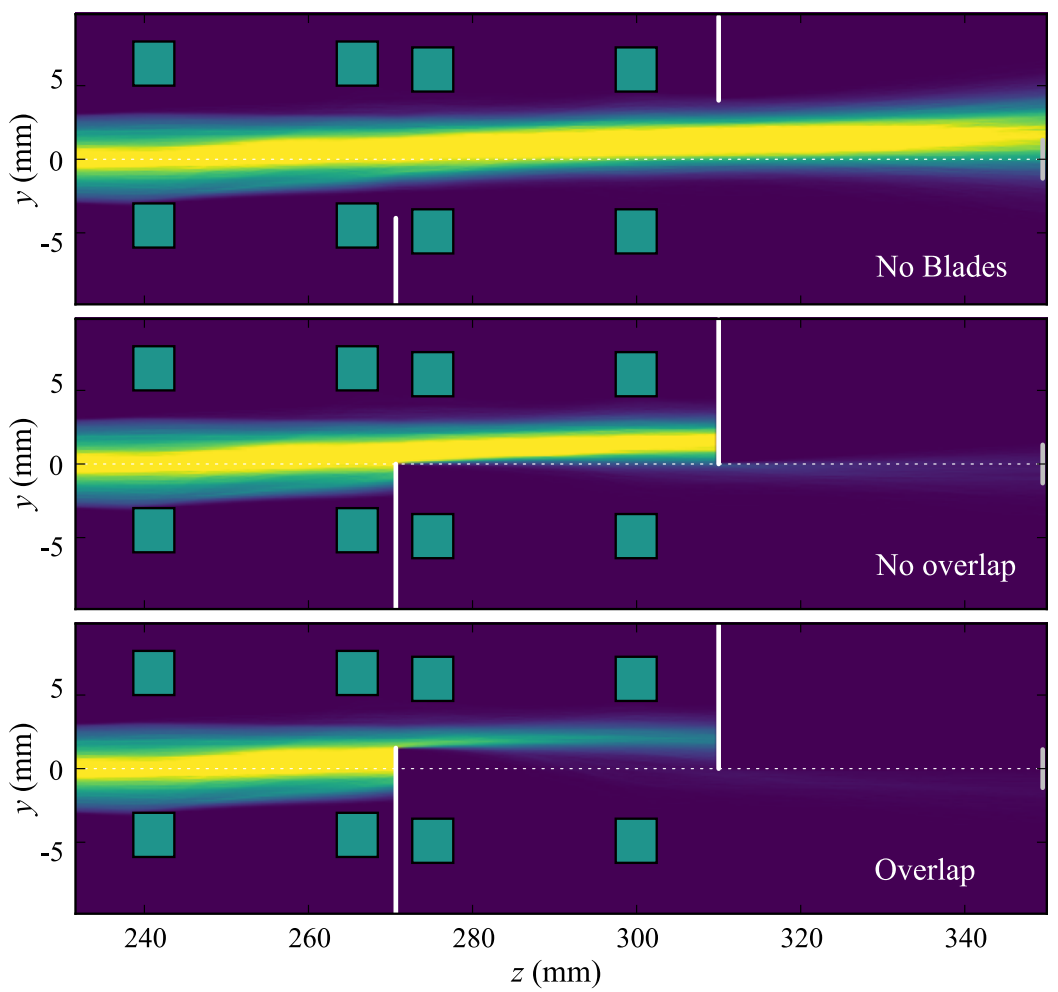

FIG. 10. Three dimensional numerical particle trajectory simulations of a beam of $1 \mathrm{~s}^{2} S_{1 / 2}$ hydrogen atoms flying through the guide. The incoming beam has already passed through a 12-stage Zeeman decelerator with a target final velocity of $200 \mathrm{~ms}^{-1}$. The trajectories of particles travelling faster than the target species $\left(v_{z}>210 \mathrm{~ms}^{-1}\right)$ representing the majority of the beam-are shown as they travel through the magnetic guide in three different configurations, with intensity ranging from low (purple) to high (yellow). The green rectangles represent the Halbach arrays in cross section, the white solid lines represent the blades, and the gray line at $z=350 \mathrm{~mm}$ represents the detection region. The dotted horizontal white line shows the decelerator axis ( $z$ axis) on which the beam is initially centred. In the top panel, the blades are withdrawn $(b 1=-4 \mathrm{~mm}, b 2$ $=+4 \mathrm{~mm}$ ) and most of the particles travelling outside the target velocity range pass through the guide unhindered. In the middle panel, the blades extend to the beam axis $(b 1=b 2=0.0 \mathrm{~mm})$ and most of the faster-moving particles are blocked. Finally, in the bottom panel, the blades are positioned optimally $(b 1=+1.4 \mathrm{~mm}, b 2=0.0 \mathrm{~mm})$ and only the hydrogen atoms travelling within the narrow target velocity range are directed into the detection region; the vast majority of faster-moving particles are blocked. 


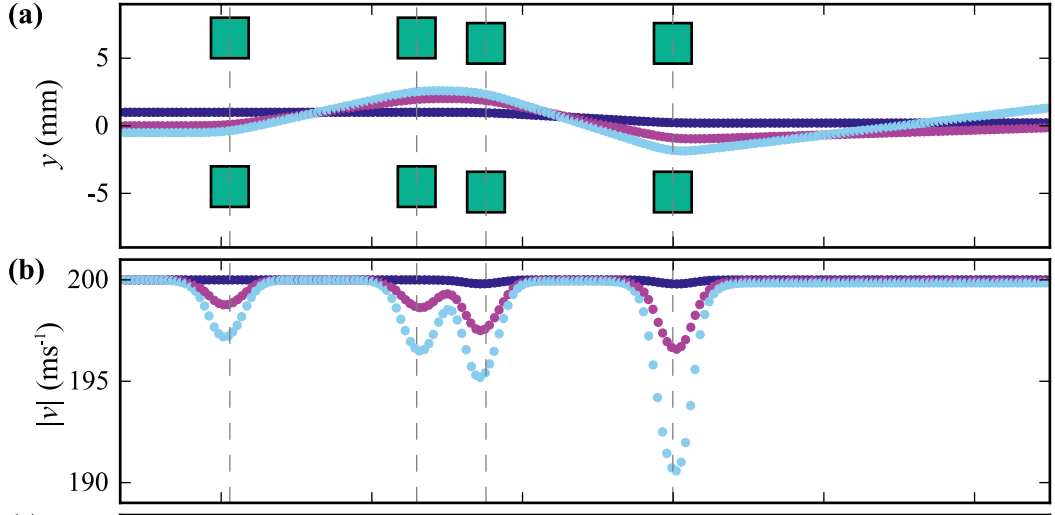

(c)
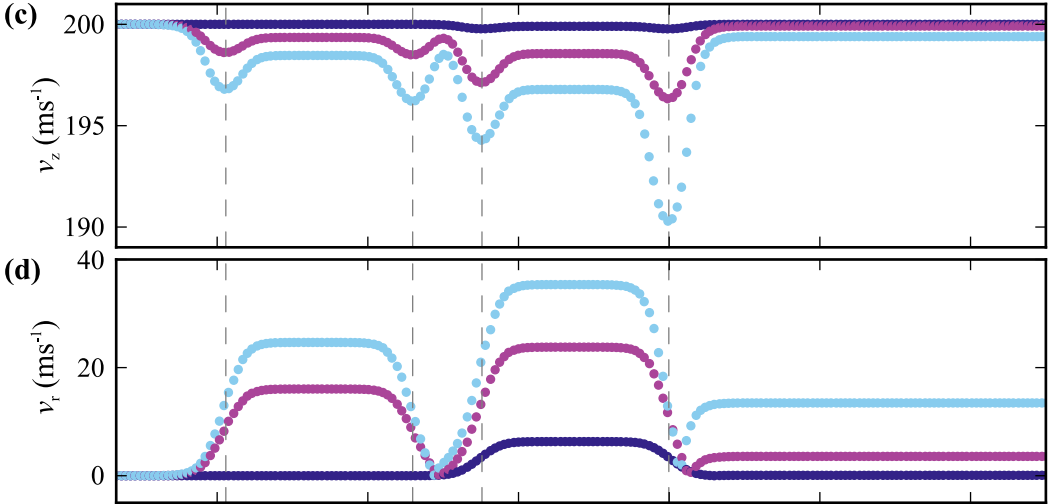

(e)

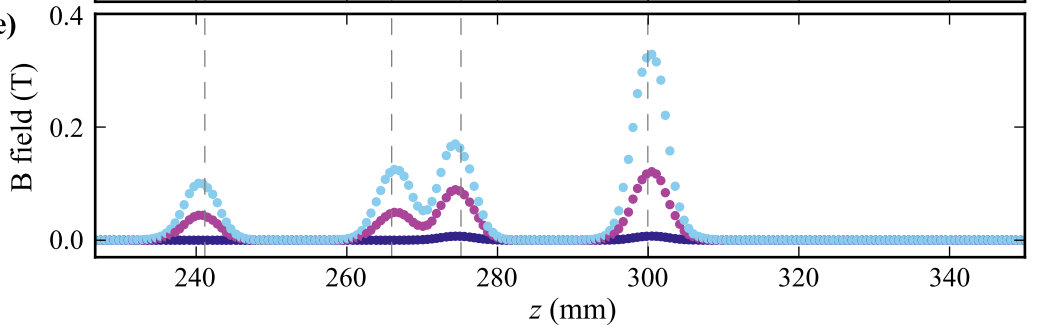

FIG. 11. (a) Simulated trajectories of three hypothetical hydrogen atoms travelling through the guide, with the green rectangles representing the Halbach arrays in cross section. The particles are initially located at $x=0.0 \mathrm{~mm}$ (all) and $y=-0.5 \mathrm{~mm}$ (light blue), $y=0.0 \mathrm{~mm}$ (purple), and $y=1.0 \mathrm{~mm}$ (dark blue), with velocity $v_{x}=v_{y}=0.0 \mathrm{~ms}^{-1}$ and $v_{z}=200 \mathrm{~ms}^{-1}$ (all). The (b) speed, (c) axial, and (d) radial velocities of the particles, as well as the (e) magnetic field they experience, are also plotted as a function of $z$ position. Dashed vertical lines indicate the centre of each array. due to the removal of particles travelling outside the desired velocity range. $^{13}$

One clear feature in Fig. 9 is the delay in the arrival time of the decelerated peak when the guide is in place. The decelerated peak is centred at approximately $1120 \mu \mathrm{s}$ when there are no Halbach arrays present. Once the four arrays comprising the magnetic guide are in place, the decelerated peak shifts to be centred at around $1160 \mu \mathrm{s}$ (irrespective of the presence of the blades). The longer flight time does not imply an overall velocity decrease: there is no point of magnetic field discontinuity with the permanent magnets in the guide (i.e., the magnetic fields are not rapidly switched as occurs in the Zeeman decelerator coils), and so there can be no net loss of kinetic energy from the beam as it passes through the guide. Particles are deflected as they travel through the guide (see Fig. 11), which results in a slightly longer flight path and an increase in their radial velocity, $v_{r}$. While there is a very minor reduction in $v_{z}$ for some particles exiting the guide, this is accompanied by an increase in their radial velocity as dictated by $v_{z}^{2}=2 E / m-v_{r}^{2}$. These effects can be clearly seen in Fig. 11, where the changes in $y$ position, $v_{z}$ and $v_{r}$ are shown for three simulated particles as they traverse the guide. As the arrays are centred on $x=0$, particles experience forces that are symmetric about the $x$ axis. This results in focusing of the beam between the first pair and between the second pair of arrays; there is no net displacement of the beam from the $x$ axis (not shown).

\section{GUIDING DIFFERENT VELOCITIES}

Hydrogen atoms travelling at different final velocities can be transmitted by simply varying the positions of the Halbach arrays within the guide. As can be seen in Fig. 8, the distance between the midpoints of the first and second (and third and fourth) HAs is $2 f$, where $f$ is the focal length. For a fixed magnetic guide assembly, the focal length depends on the square of the target beam velocity and can be derived from Eq. (2),

$$
f=-\frac{1}{\boldsymbol{M}_{21}}=\frac{1}{\lambda \sin (L \lambda)} \stackrel{\lambda L \rightarrow 0}{=} \frac{1}{L \lambda^{2}} \approx \frac{m v_{z}^{2}}{2 \mu} \frac{r_{0}^{2}}{B_{r} l_{h}} .
$$


Thus upon decreasing the focal length, the target velocity-that is, the velocity that is preferentially transmitted through the guideis decreased accordingly. As a result, translating the position of the second and fourth HAs along the $z$ axis enables a range of different target velocities to be selected. This has been demonstrated experimentally with hydrogen atoms travelling at target velocities of $200 \mathrm{~ms}^{-1}, 175 \mathrm{~ms}^{-1}, 150 \mathrm{~ms}^{-1}$, and $125 \mathrm{~ms}^{-1}$ successfully filtered from all other components of the respective Zeeman-decelerated beams using the magnetic guide. ${ }^{13}$ The simulated trajectories taken by particles travelling at lower target velocities closely resemble the behavior of the $\mathrm{H}$ atoms travelling at $200 \mathrm{~ms}^{-1}$.

The simulated percentage of $\mathrm{H}$ atoms in the guided beam travelling at the target velocity is consistently high across the 125$200 \mathrm{~ms}^{-1}$ operational range of the guide (see Fig. 12). Crucially, the guided beams also display consistently narrow velocity distributions across the entire range of target velocities. The transmission efficiency of the guide is dependent on the target velocity: $52 \%$ of particles with $v_{z}$ within $\pm 10 \mathrm{~ms}^{-1}$ of the target velocity are transmitted for $v_{z}=200 \mathrm{~ms}^{-1}$ and $175 \mathrm{~ms}^{-1}$, with the transmission efficiency dropping to $42 \%$ for $150 \mathrm{~ms}^{-1}$ and $22 \%$ for $125 \mathrm{~ms}^{-1}$. The decrease in the percentage of particles transmitted through the guide as the target velocity is lowered is due to the selected guide geometry $(s 12, s 34$, $b 1, b 2$ ), which is optimised for the target velocity of $200 \mathrm{~ms}^{-1}$. Only the distance between the HAs, $2 f$ (see Fig. 8), is modified when guiding lower final velocities. Optimising other adjustable parameters of the guide (such as the extent to which each pair of HAs is shifted,
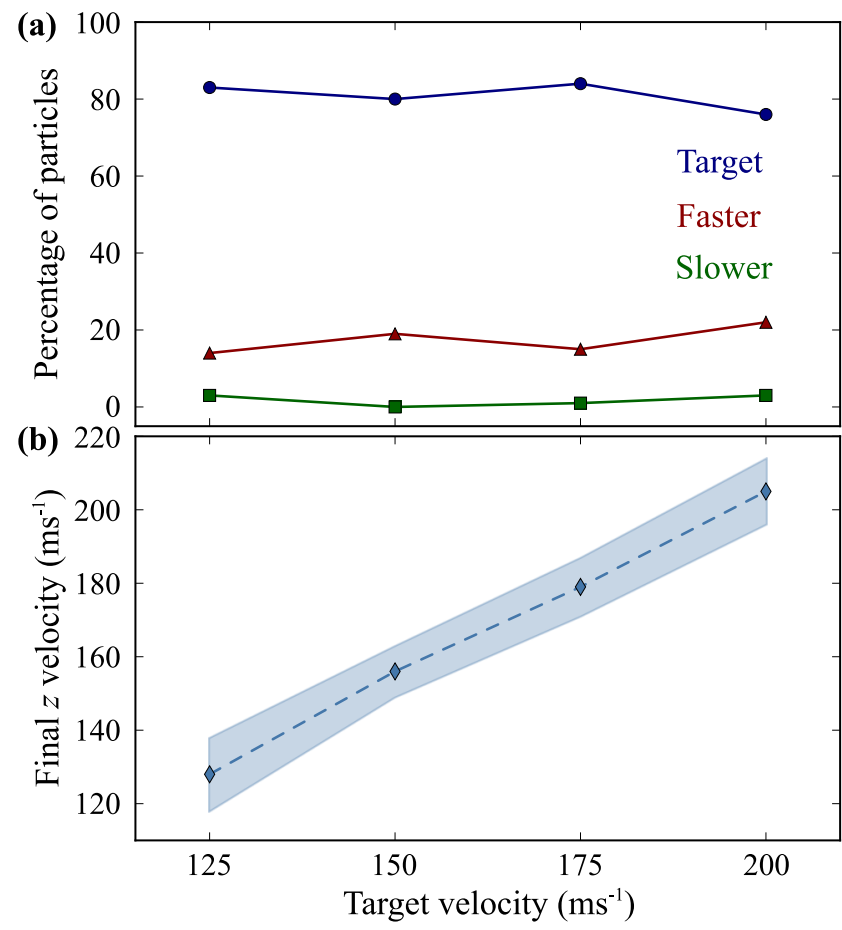

FIG. 12. (a) Simulated percentage of particles with target (blue circles), faster (red triangles), and slower (green squares) velocities emerging from the guide for each target velocity. (b) Average velocity of the particles transmitted through the guide for each target velocity (light blue diamonds), with the shaded area representing one standard deviation. $s 12$ and s34) for each individual target velocity is anticipated to significantly improve the transmission efficiency at the lower target velocities.

\section{CONCLUSION}

Hydrogen atoms can be straightforwardly manipulated with a single tilted or shifted Halbach array. By quantifying the amount of deflection achievable with one HA, it is established that a combination of four shifted HAs and two skimming blades will act as an effective beam filter-transmitting only the radical species of interest. This is successfully demonstrated experimentally with $\mathrm{H}$ atoms travelling at a selected velocity exclusively guided through the series of HAs and around the skimming blades into the detection region. The positions of the HAs and the skimming blades within the magnetic guide can be adjusted to maximise the number of hydrogen atoms transmitted or minimise the velocity distribution of the resulting beam, for velocities falling within the range $125-200 \mathrm{~ms}^{-1}$. Should one wish to generate a pure, velocity-selected beam of $\mathrm{H}$ atoms travelling at higher velocities or to target a different radical species such as $\mathrm{O}$ atoms, the Halbach arrays described in this work could be switched for arrays with different properties (thickness, radii, and magnetisation).

\section{ACKNOWLEDGMENTS}

B.R.H. acknowledges the financial support of the EPSRC (Project Nos. EP/N004647/1 and EP/N032950/1). J.T. acknowledges the support of the EPSRC in the form of a Doctoral Prize award and Merton College for a Simms Bursary. Supporting data can be obtained from the Oxford Research Archive (https://doi.org/ 10.5287/bodleian:ozVBnYNpB).

\section{REFERENCES}

${ }^{1}$ J. Thiebaud, A. Aluculesei, and C. Fittschen, J. Chem. Phys. 126, 186101 (2007).

${ }^{2}$ L. Ghassemzadeh, T. J. Peckham, T. Weissbach, X. Luo, and S. Holdcroft, J. Am. Chem. Soc. 135, 15923 (2013).

${ }^{3}$ J. Slevin and W. Stirling, Rev. Sci. Instrum. 52, 1780 (1981).

${ }^{4}$ G. Herzberg, Faraday Discuss. Chem. Soc. 71, 165 (1981).

${ }^{5}$ K. Hayashi, N. Sakudo, T. Noda, A. Takeda, K. Fujimura, and N. Shimizu, Nucl, Instrum. Methods Phys. Res., Sect. B 127-128, 918 (1997).

${ }^{6}$ A. W. Harrison, M. Ryazanov, E. N. Sullivan, and D. M. Neumark, J. Chem. Phys. 145, 024305 (2016).

${ }^{7}$ T. Cremers, S. Chefdeville, N. Janssen, E. Sweers, S. Koot, P. Claus, and S. Y. T. van de Meerakker, Phys. Rev. A 95, 043415 (2017).

${ }^{8}$ Y. Arimoto, T. Yoshioka, H. M. Shimizu, K. Mishima, T. Ino, K. Taketani, S. Muto, M. Kitaguchi, S. Imajo, Y. Iwashita et al., Phys. Procedia 17, 20 (2011).

${ }^{9}$ S. Truppe, H. J. Williams, N. J. Fitch, M. Hambach, T. E. Wall, E. A. Hinds, B. E. Sauer, and M. R. Tarbutt, New J. Phys. 19, 022001 (2017).

${ }^{10}$ K. Dulitz, M. Motsch, N. Vanhaecke, and T. P. Softley, J. Chem. Phys. 140, 104201 (2014).

${ }^{11}$ J. Toscano, A. Tauschinsky, K. Dulitz, C. J. Rennick, B. R. Heazlewood, and T. P. Softley, New J. Phys. 19, 083016 (2017).

${ }^{12}$ K. Dulitz and T. P. Softley, Eur. Phys. J. D 70, 19 (2016).

${ }^{13}$ J. Toscano, C. J. Rennick, T. P. Softley, and B. R. Heazlewood, J. Chem. Phys. 149, 174201 (2018).

${ }^{14}$ K. Halbach, Nucl. Instrum. Methods 169, 1 (1980).

${ }^{15}$ J. P. Beardmore, A. J. Palmer, K. C. Kuiper, and R. T. Sang, Rev. Sci. Instrum. 80, 073105 (2009). 
${ }^{16} \mathrm{P}$. Elleaume, O. Chubar, and J. Chavanne, in Proceedings of the 1997 Particle Accelerator Conference (IEEE, Vancouver, B.C., Canada, 1997), Vol. 3, pp. 35093511, ISBN: 0-7803-4376-X, URL http://accelconf.web.cern.ch/accelconf/pac97/ papers/pdf/9P027.PDF.

${ }^{17}$ O. Chubar, P. Elleaume, and J. Chavanne, J. Synchrotron Radiat. 5, 481 (2007).

${ }^{18}$ J. Chavanne, Radia (version 4.29), 2009, URL http://www.esrf.eu/Accelerators/ Groups/InsertionDevices/Software/Radia.
${ }^{19}$ G. Borodi, Ph.D. thesis, Chemnitz University of Technology, Chemnitz, 2008.

${ }^{20}$ A. E. Siegman, Lasers (University Science Books, Mill Valley, California, 1986), ISBN: 978-0-935702-11-8; 978-0-19-855713-5, oCLC: 14525287.

${ }^{21}$ Note that the influence of a free flight region between the beam source and the HA was neglected as its contribution is small, thanks to the low divergence of the beam. 